
allemande

45-2 | 2013

Images et discours de la nation

\title{
Nation et arts plastiques en RDA
}

Jacques Poumet

\section{OpenEdition}

\section{Journals}

Édition électronique

URL : https://journals.openedition.org/allemagne/1507

DOI : 10.4000/allemagne.1507

ISSN : 2605-7913

Éditeur

Société d'études allemandes

\section{Édition imprimée}

Date de publication : 30 décembre 2013

Pagination : 321-338

ISSN : 0035-0974

\section{Référence électronique}

Jacques Poumet, "Nation et arts plastiques en RDA », Revue d'Allemagne et des pays de lanque allemande [En ligne], 45-2 | 2013, mis en ligne le 29 juillet 2019, consulté le 23 mai 2021. URL : http:// journals.openedition.org/allemagne/1507 ; DOI : https://doi.org/10.4000/allemagne.1507 


\section{Nation et arts plastiques en RDA}

\section{- Jacques Poumet*}

Dans les années 1950 et 1960, la RDA a conservé officiellement la référence à l'unité de la nation allemande. Dans la constitution de 1968, l'intérêt supérieur de la nation allemande est invoqué comme élément de légitimation d'une RDA définie comme un «État socialiste de nation allemande ». Au début des années 70, la RDA abandonne progressivement la référence à la nation allemande et se réclame d'une autre nation, la nation socialiste. Dès le VIII e congrès du SED en 1971, il est question de la " nation socialiste » qui se développe dans l'« État socialiste allemand » de RDA, par opposition à la nation bourgeoise qui se perpétue en Allemagne de l'Ouest ${ }^{(1)}$. L'année suivante, les choses se précisent: l'Allemagne divisée, ce ne sont pas deux États d'une même nation, mais deux nations incarnées dans des États aux régimes sociaux différents ${ }^{(2)}$. En 1973, les commentaires officiels martèlent l'idée que le processus de démarcation (Abgrenzung) entre les deux États traverse également la nation et qu'aucune communauté linguistique, culturelle, ou historique entre les populations des deux Allemagnes ne saurait fonder l'existence présente d'une nation commune ${ }^{(3)}$. Une fois acquis le traité fondamental entre les deux Allemagnes et la reconnaissance internationale de la RDA,

* Professeur émérite, Université Lumière Lyon 2.

1 "Im Gegensatz zur BRD, wo die bürgerliche Nation fortbesteht [...], entwickelt sich bei uns in der Deutschen Demokratischen Republik, im sozialistischen deutschen Staat, die sozialistische Nation. » Bericht des Zentralkomitees an den VIII. Parteitag der Sozialistischen Einheitspartei Deutschlands, Berlin, Dietz Verlag, 1971, p. 56.

2 Albert Norden, Fragen des Kampfes gegen den Imperialismus, Vortrag an der Parteihochschule KarlMarx, 03.07.1972, Berlin, 1972, p. 22. Cité dans: Bundesministerium für innerdeutsche Beziehungen (éd.), DDR-Handbuch, Cologne, Verlag Wissenschaft und Politik, 1985, p. 924. Cf. aussi: Carl Christoph Schweitzer (dir.), Die deutsche Nation. Aussagen von Bismarck bis Honecker, Cologne, Verlag Wissenschaft u. Politik, 1976.

3 "Zwischen der sozialistischen Nation in der DDR und der kapitalistischen Nation in der BRD hat sich [...] die historische Tendenz der Abgrenzung durchgesetzt. » Albert Norden, Neues Deutschland, 20.03.1973. 
ce processus est ancré dans la constitution en 1974. Toute référence à l'Allemagne et à la nation allemande disparaît de la constitution, et la RDA se définit désormais comme « un État socialiste des ouvriers et des paysans » : la nation allemande est réputée appartenir au passé, la perspective de la réunification n'est plus mentionnée. La formule « deux États, une Nation » qui sous-tend en République fédérale la nouvelle politique à l'Est relève dans cette perspective d'un aveuglement historique, ou d'une volonté de renverser le cours de l'Histoire.

Ce remplacement de la nation allemande par la nation socialiste se heurtera à l'incompréhension d'une grande partie de la population, qui s'identifie jusqu'à un certain point avec la RDA sans s'identifier pour autant à une nation socialiste internationale, et qui ne perd pas la conscience d'appartenir à un ensemble allemand. Pour répondre à cette incompréhension, la notion de nation socialiste sera complétée par celle de « nationalité allemande » qui désigne l'appartenance non pas à une nation, mais à un groupe linguistique, culturel et ethnique ${ }^{(4)}$. La position officielle sera assouplie dans la pratique au cours des dix années suivantes, et on verra resurgir au moment de la crise des fusées Pershing et SS20 l'évocation du «peuple allemand » au nom duquel la RDA s'associe à la revendication d'une zone dénucléarisée au centre de l'Europe (1983). Les commentaires autorisés n'excluent plus l'avènement lointain d'une « nation socialiste allemande " qui verrait le jour quand le capitalisme aurait disparu dans toute l'Allemagne ${ }^{(5)}$. La théorie des deux nations - nation des Krupp à l'Ouest, nation des Bebel et des Liebknecht à l'Est - n'est pas officiellement abandonnée, mais dans la pratique elle n'est plus utilisée. D’autant moins qu'avec la montée en puissance de Solidarnosc en Pologne et le désaccord fondamental avec la politique de Perestroika menée en Union soviétique, la conscience d'une nation socialiste internationale n'était plus à l'ordre du jour en RDA.

La conception changeante de la nation se répercute sur la définition des rapports entre culture et nation. Au plus fort de la guerre froide, dans les années 1950, la RDA revendique l'ensemble de l'héritage culturel progressiste allemand, tout l'héritage positif de la nation allemande. La culture est du côté de la RDA, en République fédérale règne l'inculture, la décadence, la barbarie culturelle (Kulturbarbarei) : la culture allemande étant indivisible, la RDA se présentait comme seule héritière possible de la culture nationale. La République fédérale, enfant illégitime, n’héritait de rien. À la première conférence de Bitterfeld qui lance en 1959 le mouvement des écrivainsouvriers, Walter Ulbricht définit l'objectif de la manière suivante: «Wir wollen [...] die Kultur des neuen Deutschlands gestalten, jene Kultur, die ihrer Form nach national und ihrem Inhalt nach eine sozialistische Kultur ist ${ }^{(6)}$. Cette question de la forme

4 Bericht des Politbüros an die 13. Tagung des ZK der SED, Neues Deutschland, 13.12.1974. La formule employée par Erich Honecker était: «Staatsbürgerschaft: DDR, Nationalität: Deutsch ».

5 «Die Frage, ob in späterer Zeit, wenn die Arbeiterklasse der BRD im Bündnis mit allen Werktätigen die sozialistische Umgestaltung der Gesellschaft und der kapitalistischen Nation erkämpft haben wird, eine einheitliche sozialistische deutsche Nation entstehen kann, muß gegenwärtig offen bleiben ». Kleines politisches Wörterbuch, Berlin, Dietz Verlag, 1983.

6 Walter Ulbricht, Schlußwort auf der 1. Bitterfelder Konferenz. Cité dans: Klaus Jarmatz (dir.), Kritik in der Zeit, Halle/Saale, Mitteldeutscher Verlag, 1970, p. 461. 
nationale renvoie d'une part à la culture d'une Allemagne conçue comme unité: à une époque où le mur de Berlin n'est pas encore construit et où la réunification reste l'objectif officiel, les formes de culture développées en RDA sont conçues comme ayant vocation à devenir celles de toute l'Allemagne. La forme nationale signifie d'autre part que cette nouvelle culture est développée dans un cadre national, mais qu'elle a une vocation universelle en tant qu'expression de la société socialiste. Est « nationale » la référence aux classiques qu'on doit rendre accessible à tous, est « internationale " ou universelle la représentation de la nouvelle qualité des rapports sociaux.

Une fois la division allemande durablement établie par la construction du mur de Berlin, la RDA adapte son discours sur la culture nationale. La tradition culturelle est coupée en deux: la RDA est désormais héritière de la tradition progressiste, la République fédérale est héritière de la tradition réactionnaire. Thomas Müntzer fait partie d'emblée de cette tradition progressiste, mais Luther en est largement exclu: en tant que réformateur en lutte contre la papauté il trouve place dans la tradition nationale, mais en tant que défenseur des princes contre les paysans il en est rejeté: c'est une position équilibriste difficile à tenir. Il n'y a plus de culture allemande indivisible, mais une culture nationale spécifique de la RDA, la « culture nationale socialiste » (sozialistische Nationalkultur) ${ }^{(7)}$.

Dans les années 1980, confrontée à un déficit de légitimation, la RDA réintègre dans son Panthéon des pans entiers de l'histoire nationale allemande auxquels elle se déclarait jusqu'alors étrangère: la Prusse et Frédéric II à l'occasion de l'année de la Prusse en 1980, Luther à l'occasion du 500 e anniversaire de sa naissance en 1983, Bismarck lui-même malgré sa lutte acharnée contre les socialistes. Il est apparu qu'il y a des lieux de mémoire interallemands, et que l'on ne pouvait pas fonder une conscience nationale en RDA en excluant de grandes figures qui sont des références identitaires à l'Est comme à l'Ouest. On ne peut pas fonder un sentiment d'identité nationale sans Frédéric II, Luther, Bismarck.

Si on prend au mot la théorie officielle sur la nation socialiste, toutes les représentations picturales du mouvement ouvrier avant 1949 sont des représentations de la nation en devenir, et toutes les représentations de la place de l'ouvrier dans la société et dans l'État illustrent de facto la réalisation de cette nation. Ces deux types de représentations sont légion dans les arts plastiques de RDA. Révolutions, manifestations, répressions du passé peuplent une peinture historique abondante, et le monde de l'entreprise, avec ses rapports sociaux nouveaux, est omniprésent dans la peinture du quotidien: les discussions sur le lieu de travail, l'importance du collectif et de la brigade, la fierté ouvrière, le travail sous toutes ses formes, la maîtrise du progrès technique. Les sujets historiques sont autant d'occasions d'évoquer la tradition dont se réclame la RDA et les traumatismes de l'histoire allemande. Les commandes institutionnelles qui sont un volet important de l'économie de l'art en RDA et contribuent pour une bonne part au financement de la création artistique font une large place aux représentations des faits marquants de

7 « Es ist eine eigenständige sozialistische Nationalkultur der DDR entstanden. Diese Kultur ist Teil der sich entwickelnden sozialistischen Weltkultur. » Kurt Hager, Schlußwort auf der zentralen Parteiaktivtagung im Kulturbund der DDR in Berlin, 19 Juni 1980, Sonntag, 29. Juni 1980. Cité par: Matthias JuDT (dir.), DDR-Geschichte in Dokumenten, Bonn, Bundeszentrale für politische Bildung, 1998, p. 335. 
l'évolution du mouvement ouvrier en Allemagne et dans le monde, aux bouleversements de l'histoire moderne (guerre des paysans, guerre de trente ans), aux épisodes de l'histoire contemporaine devenus lieux de mémoire (guerres de libération, révolution de $1848)^{(8)}$. Les expositions quinquennales de peinture et d'arts graphiques organisées au niveau des districts et au niveau national affichent volontiers des œuvres en rapport avec les commémorations les plus actuelles (p. ex. centenaire de la Commune de Paris).

Werner Tübke, Frühbürgerliche Revolution in Deutschland 1525 (1983-1987)

(détail: Champ de bataille), 14x123m, Panoramamuseum Bad Frankenhausen. @Adagp, Paris, 2013.

Werner Tübke a peint la fresque circulaire monumentale du mémorial de Bad Frankenhausen, édifié sur le lieu de la bataille qui conduisit à la défaite de Thomas Müntzer dans la guerre des paysans, le «Schlachtberg ». Tübke avait été chargé de réaliser cette fresque à l'occasion du $450^{\mathrm{e}}$ anniversaire de la guerre des paysans, en 1975, et il y a travaillé plus de dix ans jusqu'à son inauguration en septembre 1989, pour le $500^{\mathrm{e}}$ anniversaire de la mort de Thomas Müntzer, les quarante ans de la RDA, et... deux mois avant la chute du mur. Ce panorama a été voulu comme une ouvre d'importance nationale, c'est une œuvre de commande qui doit manifester la place éminente qu'occupe Thomas Müntzer dans l'histoire nationale telle que la lit la RDA. Il s'agit en principe d'illustrer le thème de la " première révolution bourgeoise » (frühbürgerliche Revolution) qui, dans l'interprétation marxiste, préfigure la révolution prolétarienne future: le mouvement radical de la Réforme, qui s'élève contre le pouvoir féodal, est lu comme le premier acte d'une tradition révolutionnaire nationale en Allemagne qui, à travers la révolution de 1848 et celle de novembre 1918, aboutit à la fondation de la RDA. De nombreuses

8 Sur la fonction des commandes institutionnelles, cf. Jérome BAZIN, « Réalisme et égalité. Contribution à une histoire sociale de la peinture et des arts graphiques en République Démocratique Allemande (1949-1990) », thèse de doctorat, Université d'Amiens / Université de Genève, 2011, p. 87-133. 
œuvres ont été consacrées à ce thème dans les années 1950 et $1970^{(9)}$. Mais c'est une tout autre interprétation que livre Tübke: l'extrait ci-contre représente la bataille au milieu de laquelle se trouve Thomas Müntzer, l'air découragé, son drapeau posé à terre en signe de défaite. L'ensemble de cette œuvre circulaire est organisé en quatre parties: printemps, été, automne, hiver. La circularité des saisons ne débouche sur aucune perspective d'avenir, la rotondité même de la fresque induit l'impression d'un perpétuel recommencement du même. L'idée d'un progrès en histoire est abandonnée au profit d'une représentation foisonnante et chaotique - et cela à quelques semaines de la fin de la $\mathrm{RDA}^{(10)}$. Le peintre en est lui-même conscient, et il plaide non sans un brin de provocation pour une interprétation de son œuvre en fonction de critères purement esthétiques faisant abstraction des considérations historiques ${ }^{(11)}$.

La confrontation avec le passé national-socialiste occupe une place éminente dans le paradigme des représentations du passé national dans la peinture de RDA. En République fédérale, l'héritage du national-socialisme a été très présent dans le débat public des années 1970, et la Shoah a fait l'objet d'un travail de mémoire intense dans les années 1980. Les arts plastiques sont le théâtre et l'instrument d'une recherche obsédante, celle des traces de la génération des pères compromis avec le nazisme. Les symboles nazis, les uniformes de la Wehrmacht, les photos de famille datant du troisième Reich sont intégrés dans l'image et renvoient au refoulement de l'histoire allemande (Gerhard Richter, Markus Lüperz, Anselm Kiefer). Dans la série « Café Deutschland » de Jörg Immendorf, les fantômes du passé nazi sont également à l'affût derrière le décor d'un bar contemporain. Cette recherche se poursuit au cours des années 1980 dans toutes les formes des arts plastiques et engendre des œuvres parfois spectaculaires dans lesquelles de gigantesques croix gammées surgissent des bâtiments anciens et de l'environnement quotidien (Albert Oehlen, Martin Kippenberger, Olaf Metzel).

Souvent désigné comme le mythe fondateur de la RDA, l'antifascisme a été un élément central de légitimation pour le SED et a rencontré un écho favorable dans la population. Reposant sur une analyse qui assimilait le nazisme et le fascisme à une dictature du grand capital, il affirmait que la RDA, rompant avec le capitalisme, avait extirpé chez elle les racines du fascisme. Le rappel constant de la résistance contre le nazisme faisait du SED l'héritier d'une lutte héroïque, et de la RDA tout entière l'État antifasciste par excellence, exempt de tout lien de filiation avec le nazisme et

9 Dans les années 1950, on peut citer en particulier Max Lingner "Großer deutscher Bauernkrieg " (1955), Lea Grundig "Zum deutschen Bauernkrieg » (1956), Bert Heller « Scheideweg XX. Jahrhundert ». Pour les années 1970, le triptyque de Heinz Zander (1971), ainsi que les tableaux de Horst Sakulowski « Deutschland 1525 - Die Auferstehung ", et ceux de Willi Neubert, Volker Stelzmann, Ronald Paris. Cf. à ce sujet Rudolf KoBER, « Revolution im Bild », UZ /03, 19. + 26. Januar 1990 (Universitätszeitung, Leipzig).

10 Eckhart Gillen, Feindliche Brüder? Der kalte Krieg und die deutsche Kunst 1945-1990, Berlin, Nicolai, 2009, p. 426-428; Stéphanie Barron, Sabine Eckmann (dir.), Kunst und kalter Krieg. Deutsche Positionen 1945-89, Cologne, DuMont, 2009, p. 350.

11 Bärbel Mann, Jörn Schütrumpf, «Werner Tübke: Frühbürgerliche Revolution in Deutschland, Panorama auf dem Schlachtberg bei Bad Frankenhausen ", in: Monika Flacke (dir.), Auftrag Kunst 1949-1989. Bildende Künstler der DDR zwischen Ästhetik und Politik, Berlin, Deutsches Historisches Museum, 1995, p. 377. 
étranger au fascisme allemand dont il ne partageait aucunement la responsabilité. Au côté de l'Union soviétique qui avait contribué de façon décisive au renversement de la dictature nazie, on était dans le camp des "vainqueurs de l'histoire ». Cette formule permettait de renvoyer l'héritage de la culpabilité dans le camp adverse et de disculper la population de RDA, dispensée de s'interroger sur le soutien qu'une large partie de la population allemande avait apporté au régime nazi. Dans son rapport pour la commission d'enquête parlementaire sur « l'histoire et les conséquences de la dictature du SED en Allemagne ", l'historien Bernd Faulenbach a résumé ce phénomène en écrivant: «L'héritage national-socialiste est devenu le problème des Allemands de l'Ouest. Hitler était devenu pour ainsi dire un Allemand de l'Ouest » ${ }^{(12)}$.

En RDA, les peintres de "L'école de Leipzig " ont accordé une place importante au passé national-socialiste et à son refoulement. Dès les années 1960, Heisig évoque directement son expérience personnelle, la bataille de Breslau qu'il a vécue comme adolescent engagé dans la Waffen-SS pour défendre la ville. Son expérience de la guerre et du nazisme hante son œuvre. C'est un des représentants les plus importants de la peinture historique en RDA. Son ouvre est obsédée par le " cauchemar nazi ». Ses tableaux les plus connus se présentent comme un enchevêtrement de fragments extraits de l'univers contemporain et de fragments empruntés à l'horreur nazie. Le " cauchemar fasciste " est représenté comme une folie chaotique, une orgie de violence meurtrière. L'évocation de la défense forcenée de Breslau dans «Festung Breslau - Die Stadt und ihre Mörder " (1968) est une vision apocalyptique dans laquelle on reconnaît pêle-mêle le pont qui symbolise la ville (Dombrücke), un soldat qui se voile la face avec son casque, une femme dénudée qui symbolise la profanation de la ville, un dignitaire nazi qui hurle, des haut-parleurs de la propagande, un adolescent qui tire au fusil mitrailleur (et qui représente évidemment le peintre), un char en feu d'où un soldat s'échappe, etc. Il existe une variante de ce tableau peinte en 1973 et intitulée « Unterm Hakenkreuz ». Heisig poursuit sans interruption ce travail sur son expérience personnelle jusqu'à la fin des années 1980 .

Dans la décennie suivante, il représente une « obstination de l'oubli » («Beharrlichkeit des Vergessens », 1977) dans laquelle les «mécanismes de l'oubli » (pour reprendre le titre d'un autre tableau) transcendent la frontière entre les systèmes: ce sont des mécanismes interallemands. Les symboles modernes de la manipulation des masses, une batterie de haut-parleurs et des images de film se mêlent aux symboles anciens dans des compositions qui évoquent le passé national-socialiste comme un héritage commun aux deux Allemagnes ${ }^{(13)}$. Au centre, un mutilé de guerre allongé sur un drapeau nazi brandit son moignon et agite sa jambe de bois au rythme d'une marche militaire jouée par un bouffon. Dans sa main droite encore valide, il tient sa croix de

12 «Das NS-Erbe wurde zum Problem der Westdeutschen, Hitler war gleichsam ein Westdeutscher geworden. » Bernd Faulenbach, "Zur Funktion des Antifaschismus in der SBZ/DDR », in: I. Drechsler, B. Faulenbach, M. Gutzeit, M. Meckel, H. Weber, Getrennte Vergangenheit, gemeinsame Zukunft. Ausgewählte Dokumente, Zeitzeugenberichte und Diskussionen der Enquete-Kommission "Aufarbeitung von Geschichte und Folgen der SED-Diktatur in Deutschland » des Deutschen Bundestags 19921994, Munich, DTV, 1997, vol. 1, p. 149.

13 Jacques Poumet, "L'antifascisme de RDA et sa mise en question ", in: Catherine FAbre-Renault, Elisa Goudin, Carola Hähnel-Mesnard, La RDA au passé présent, Paris, Presses Sorbonne Nouvelle, 2006, p. 223-224. 
guerre qu'il contemple avec une expression qui tient à la fois du rire dément et du cri de douleur. Ce tableau est une des nombreuses variations peintes par Heisig sur le thème du permissionnaire poursuivi par ses cauchemars de guerre, et de la folie ordinaire de l'ancien combattant resté militariste. Les images qui assaillent le personnage central mêlent des accessoires renvoyant aux deux guerres mondiales, des fragments éclatés de la tuerie (des soldats morts, d'autres qui s'entretuent, une tête ensanglantée sans corps, un tableau d'Otto Dix, des prisonniers en cage) et, dominant le tout, un spectateur terrorisé qui dans d'autres tableaux de la même série porte les traits du peintre. Cette danse macabre est surmontée d'une banderole qui traverse le tableau comme un trait d'union entre passé et présent, entre guerres passées et menaces de guerres présentes, et sur laquelle on lit « Nous sommes pourtant tous frères et sœurs » ("Wir sind doch alle Brüder und Schwestern »). Le texte de la banderole actualise la scène et renvoie plus particulièrement à l'Allemagne de l'Ouest - la formule « frères et sœurs » ("Brüder und Schwestern ») désignait les habitants de RDA dans les discours tenus en République fédérale avant la réunification, et il dissimule en partie le cadran d'une horloge qui affiche cinq minutes après minuit - fünf nach zwölf-, l'heure des cauchemars mais aussi un avertissement aux attardés du militarisme dont le temps est révolu. Mais le contexte interallemand ne se réduit pas ici à une simple dénonciation de la République fédérale. Ce qui est désigné, c’est la fausse innocence de l'oubli, l'impossibilité d'une innocence après le nazisme (l'étreinte amoureuse côtoie le combat au corps à corps de deux soldats), particulièrement pour la génération qui l'a vécu et qui, comme Heisig, s'y est engagé activement. Il y a là une forme de mise en cause de l'externalisation du nazisme à laquelle conduit l'antifascisme tel que le conçoit et le pratique la RDA.

Bernhard Heisig, Beharrlichkeit des Vergessens (1977),

bpk/ Staatliche Museen zu Berlin, Nationalgalerie. () Adagp, Paris, 2013. 
Wolfgang Mattheuer fait partie lui aussi de la «bande des quatre » qui a fait la renommée internationale de "L'école de Leipzig ». Il présente en 1982 un tableau intitulé «Cauchemar» («Albtraum ») dont deux versions sont montrées la même année en RDA et en République fédérale. Une silhouette schématique aux membres disproportionnés traverse au pas de course un espace sombre et désert en suivant une ligne diagonale noire. Un bras dressé et

Wolfgang Mattheuer, Albtraum (1982), Kunstsammlung der Berliner Volksbank. (c) Adagp, Paris, 2013. une jambe bottée dessinent des symboles du nazisme (salut hitlérien et croix gammée), alors que l'autre bras tend un poing levé - symbole du mouvement ouvrier - et l'autre jambe tendue se termine par un pied nu. La tête atrophiée, un ovale noir sans visage, indique qu'il s'agit d'un personnage générique: un personnage hybride issu pour moitié du mouvement socialiste et pour moitié du passé nazi, et qui réunit en lui ces deux composants de manière indissociable. Les deux traditions antagonistes dont l'opposition est soulignée par la couleur (noir pour l'une, blanc pour l'autre) sont réunies dans une même figure humaine sans individualité. On est loin de la " personnalité socialiste développée » dont l'idée est propagée par le régime, cet homme nouveau longtemps invoqué par le discours officiel ne peut être dans le meilleur des cas qu'un produit hybride marqué par le passé qui ne passe pas, au sens où Christa Wolf l'entend dans son roman Trame d'enfance ${ }^{(14)}$. De même que, pour Mattheuer, le socialisme tel qu'il est appliqué en RDA n'est pas encore dégagé de son passé stalinien et en restera imprégné tant que ce passé sera refoulé, l'homme socialiste et sa personnalité développée de toute part restent pour lui une utopie ${ }^{(15)}$. Il récuse cependant l'accusation de pessimisme et de noirceur que lui a attiré la tonalité sombre de son tableau: « [...] Et mon 'Pas du siècle', une vision noire? [...] Non, mes tableaux ne sont pas des rêves noirs, ce sont des images qui interrogent, des images réalistes de leur époque » ${ }^{(16)}$. Très remarquée à l'Est comme à l'Ouest, la sculpture reproduisant le personnage de ce tableau sous le titre « Le pas du siècle » («Der Jahrhundertschritt ") n'est pas reproduite dans le catalogue de l'exposition nationale de Dresde où elle est montrée en 1987, alors que le tableau original était reproduit en couverture du catalogue de l'exposition Zeitvergleich à Hambourg en $1983^{(17)}$. Cette œuvre trop

14 Christa Wolf, Kindheitsmuster, Berlin/Weimar, Aufbau-Verlag, 1978, p. 9.

15 Wolfgang Mattheuer, Äußerungen, Leipzig, Reclam-Verlag, 1990, p. $193+239$.

16 Ibid., p. 194.

17 «Im Übrigen ist seine Plastik 'Jahrhundertschritt' eines der signifikantesten Werke der ganzen Austellung. » Karl Max Koвer, « Zunahme an Expressivität und Farbkraft », Leipziger Volkszeitung, 03.12.1987. 


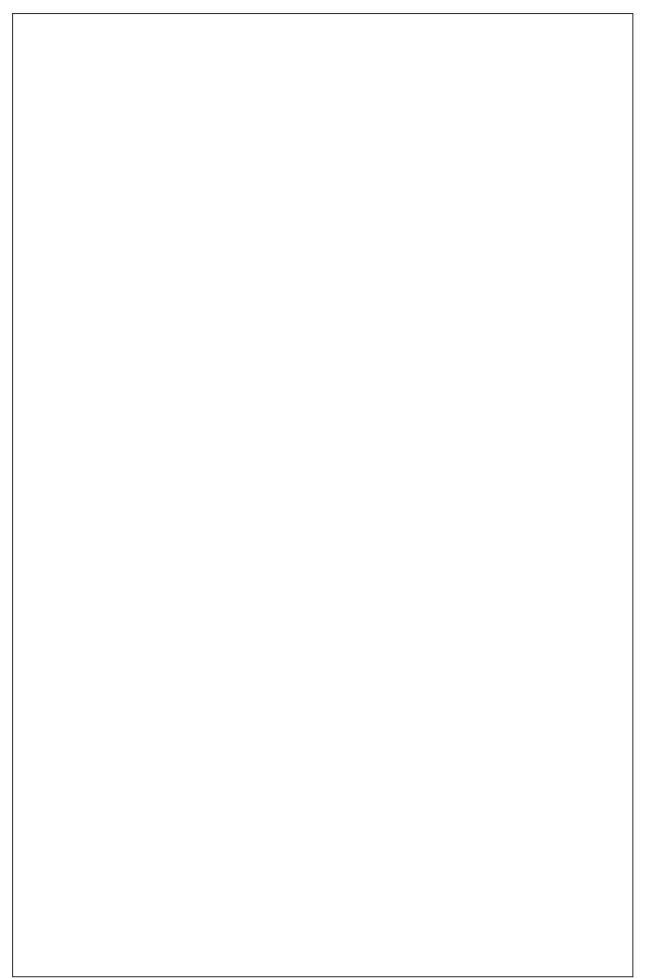

Werner Tübke, Lebenserinnerungen des Dr. jur. Schulze III (1965), bpk/ Staatliche Museen zu Berlin, Nationalgalerie. @Adagp, Paris, 2013. interallemande gêne manifestement les autorités culturelles, et peu de temps après cette dernière exposition quinquennale, Mattheuer quittera le SED dont il ne supporte plus la sclérose ${ }^{(18)}$.

L'un des tableaux majeurs de l'œuvre de Tübke s'intitule: "Souvenirs du juriste Schulze III » (« Lebenserinnerungen des Dr. jur. Schulze III ») et s'inscrit dans un cycle de sept œuvres sur le même thème peintes après le procès d'Auschwitz entre 1964 et 1967. Au centre de ce tableau monumental, le plus connu de la série, trône un juge en robe rouge, son corps est une marionnette en bois dont la tête désarticulée est tournée vers les images du passé refoulé qu'elle contemple avec complaisance. Devant le juge s'étend un paysage idyllique et harmonieux, la belle apparence d'où est évacuée toute trace des horreurs passées. Derrière lui, des scènes chaotiques de violence et de meurtre se déroulent sous le regard d'un ange du jugement dernier et d'un officier de la Wehrmacht. Les prisonniers d'un camp de concentration sont entassés comme des cadavres, un officier allemand passe la corde au coup à un condamné qui va être pendu à un arbre, des corps martyrisés aux torses nus et des soldats aux têtes de morts surplombent une pietà, et une chemise brune au brassard à croix gammée indique clairement qu'il s'agit des crimes du nazisme. La marionnette est maintenue par de multiples cordages qui plongent à la fois dans cet univers criminel et dans le socle qui porte les images d'un univers petit-bourgeois dont le juge est issu.

C'est une peinture allégorique, comme souvent chez Tübke. Le point de départ est une incitation officielle invitant les artistes à dénoncer la continuité du personnel judiciaire en République fédérale, le scandale des juges qui ont servi le nazisme et qui continuent à exercer après la guerre. Le nom passe-partout du juge "Schulze » évoque la banalité de ces reconversions qui incluent certains " juristes effrayants " (furchtbare Juristen), ceux qui ont exercé une justice sanglante au nom du régime nazi ${ }^{(19)}$. En

«Wie zu beobachten ist, löst diese Figurenkonstruktion in der Ausstellung öffentliches Nachdenken über den Charakter unserer Epoche aus. Sie bewirkt viele kontrovers geführte Debatten... » Helmut NetZKer, « Homogenität und gestalterische Kontinuität? », Bildende Kunst, 2 (1988), p. 59.

18 W. Mattheuer, Äußerungen (note 15), p. 227-228.

19 Michael Stolleis, "Furchtbare Juristen », in: Étienne François, Hagen Schulze (dir.), Deutsche Erinnerungsorte, Munich, Verlag C.H. Beck, 2001, p. 535-548. 
s'emparant de ce thème, Tübke suscite une profusion d'images de la barbarie nazie qui évoquent à la fois Breughel et Otto Dix: c'est tout le passé nazi, héritage commun des deux parties de l'Allemagne, qui resurgit dans un pêle-mêle onirique. Tübke sera bien sûr attaqué en RDA pour ce tableau dont le message est jugé ambigu, manquant de clarté, et surtout manquant d'esprit partisan, de "Parteilichkeit », et il sera à deux doigts de perdre son poste d'enseignant à l'école des Beaux-Arts de Leipzig ${ }^{(20)}$.

Hubertus Giebe, qui a été l'élève de Bernhard Heisig à l'école des Beaux-Arts de Leipzig, poursuit à travers une série de tableaux peints dans les années 1980 une recherche personnelle sur les silences et les zones d'ombre de l'antifascisme de RDA. La question qui le hante est celle de la responsabilité du stalinisme dans les déchirements fratricides du mouvement ouvrier allemand jusqu'à la Seconde Guerre mondiale. Son père, social-démocrate et antinazi, avait déserté la Wehrmacht en 1944 et était passé du côté soviétique. Mais étant social-démocrate, il était foncièrement suspect aux yeux de l'Union soviétique de Staline, et il dut faire cinq ans de camp de travail avant de pouvoir rentrer en Allemagne, en l'occurrence la RDA, à la fin de l'année $1949^{(21)}$. Après son retour, il s'était enfermé dans le silence et avait refusé jusqu'à la fin de sa vie de parler de ces années de captivité.

Dans le tableau intitulé « La Résistance (pour Peter Weiss) », Hubertus Giebe s'appuie sur une œuvre littéraire parue en République fédérale pour exhumer un pan occulté de l'histoire de la résistance communiste contre le nazisme, et pour mettre en cause

Hubertus Giebe, Der Widerstand - für Peter Weiss (1986/87), bpk/ Staatliche Museen zu Berlin, Nationalgalerie. @ Adagp, Paris, 2013.

20 E. Gillen, Feindliche Brüder? (note 10), p. 349-350.

21 Eugen Blume, Hubertus Gassner et alii, Klopfzeichen/ Wahnzimmer, Leipzig, Faber \& Faber, 2002, p. 120. 
l'antifascisme d'État en vertu duquel la RDA, rangée dans le camp des «vainqueurs de l'Histoire ", se dispense d'un retour critique sur la responsabilité de la politique stalinienne dans l'écrasement du mouvement ouvrier. En marge du tableau dominé par la répression sanglante de la résistance se tient le personnage de Willi Münzenberg, reconnaissable à son long manteau de feutre. Dans son livre L'esthétique de la Résistance (Die Ästhetik des Widerstands), Peter Weiss revenait longuement sur la biographie de ce personnage hors du commun pour en réhabiliter la mémoire. Membre du comité central du KPD depuis les années 1920, il en avait été exclu pour avoir critiqué les procès de Moscou, puis avait quitté le parti communiste et critiqué le pacte germano-soviétique. Sa mort en France en 1940 après son évasion d'un camp d'internement a été présentée comme un suicide, mais l'hypothèse d'un règlement de compte des services secrets soviétiques a souvent été avancée. La RDA lève un voile en publiant ce livre (huit ans après la parution du premier tome en République fédérale), et Hubertus Giebe trouve dans cette biographie de Münzenberg un élément de réponse à son interrogation sur la culpabilité de la génération des pères.

Le questionnement autour de l'idée de nation, présent dans la peinture qui prend pour objet l'Histoire, s'exprime également à travers les représentations métaphoriques de la dualité allemande. Les relations entre Jörg Immendorff, à l'Ouest, et A. R. Penck à l'Est sont un cas exceptionnel et concret de dialogue interallemand dans le domaine des arts plastiques. Immendorff rencontre son collègue de RDA tout d'abord dans un café de Berlin-Est, puis dans l'atelier d'A. R. Penck à Dresde. Cette coopération autour d'une interrogation commune sur le passé et le présent de la nation divisée se poursuit de la fin de l'année 1976 - qui coïncide avec l'expulsion du chanteur Wolf Biermann, déchu de la nationalité de RDA - jusqu'à l'émigration à l'Ouest d'A. R. Penck en 1980. Tous deux peignent sous des formes diverses la division de l'Allemagne et le rapport difficile avec l'idée de nation sur laquelle pèse le poids du passé nazi. Leurs rencontres sont le point de départ du cycle "Café Deutschland ", une série de 19 tableaux peints par Immendorff. Dans un cadre qui est toujours celui d'un café et d'une boîte de nuit, les deux peintres sont figurés face à face ou côte à côte, se tendant la main ou peignant ensemble, parfois représentés par de simples citations picturales. Le décor est parsemé de symboles de la division (barbelés, miradors), du passé qui ne passe pas (aigle impérial), du contexte répressif qui entoure à l'Est la poursuite de cette confrontation vivante (surveillance policière, espionnage, "démarcation " dans les mots et dans les faits).

Comme Immendorff, A. R. Penck (pseudonyme de Ralf Winkler) revendique un art politique qui invente une nouvelle façon de s'adresser au public. Le langage pictural qu'il met en œuvre depuis le début des années 1960 repose sur l'utilisation de pictogrammes anthropomorphes conçus comme un moyen d'échapper aux injonctions des idéologies de la guerre froide. Ce mode de figuration qui n'est pas sans rappeler les peintures rupestres manifeste le refus de la division Est-Ouest en peinture. Les silhouettes filiformes simplifiées à l'extrême sont dépouillées de tout trait individuel et cherchent à exprimer les attitudes fondamentales liées à la confrontation des systèmes. Elles se combinent comme des éléments d'idéogrammes pour former des caractères complexes représentant les sociétés, les activités humaines et les systèmes qui les gouvernent. 
Le tableau intitulé " le passage » (" Der Übergang », 1963) est représentatif de toute sa création et de sa position en tant qu'artiste. Un personnage schématisé franchit un abîme sur une étroite passerelle en feu. Dans une posture de funambule, les bras formant balancier et les mains largement écartées, il se tient au milieu de l'obstacle mais son mouvement le porte vers la rive d'en face. Deux ans après la construction du mur de Berlin, ce passage dangereux

A. R. Penck, Der Übergang (1963), Aachen, Ludwig Forum für Internationale Kunst. @Adagp, Paris, 2013. dans les flammes évoque la question «brûlante » de la division de l'Allemagne, l'angoisse qu'elle suscite, le libre passage désormais impossible entre les deux parties, la fragilité du lien ténu qui existe encore entre elles.

Cette façon d'utiliser des archétypes formels pour évoquer l'absurdité de la division Est-Ouest qui traverse l'Allemagne n'a pas manqué de valoir à A. R. Penck l'hostilité des autorités. Sa candidature à l'Union des Arts plastiques (VBK) est rejetée en 1969, ce qui lui ôtait la possibilité d'exposer en RDA car seuls les membres du VBK avaient accès aux expositions officielles. Pendant dix ans, il sera rejeté de fait dans l'« underground ", exposant et vendant ses tableaux en République fédérale où il s'était déjà fait un nom, mais en butte aux tracasseries administratives, à la surveillance active de la Stasi et à ses interventions directes. Il finit par émigrer à l'Ouest en 1980. Pour un peintre de Dresde originaire de cette ville, cette image du pont est en même temps un hommage chiffré au groupe « Die Brücke » fondé à Dresde dans les premières années $\mathrm{du} \mathrm{xx}^{\mathrm{e}}$ siècle, et au programme artistique et social de l'expressionnisme, c'est-à-dire à une tradition artistique commune aux deux Allemagnes.

D'autres tableaux de Penck mettent l'accent sur la confrontation Est-Ouest et l'antagonisme des deux systèmes. C’est le cas de « Weltbild 1 » (1965), dont plusieurs variantes mettent en avant tour à tour la confrontation et la réconciliation, la division et la coexistence («A.B.-Bild », 1965 / «Ohne Titel (Systembild)», 1966). Dans ces diverses versions, les figures stylisées de l'Est et de l'Ouest ne peuvent pas être distinguées les unes des autres : leur agencement évoque des sociétés différemment structurées, mais les personnages n'ont pas de caractère propre. Leurs gesticulations hostiles et leurs armes les opposent de part et d'autre d'une ligne de démarcation clairement tracée, mais la similitude des traits donne un caractère absurde à la confrontation, donne du corps à la communauté de destin nationale par-delà les images du déchirement national ${ }^{(22)}$.

22 Karen LANG, "Der Expressionismus und die beiden deutschen Staaten ", in: Barron/ECKMANN, Kunst und kalter Krieg (note 10), p. 91-92. 


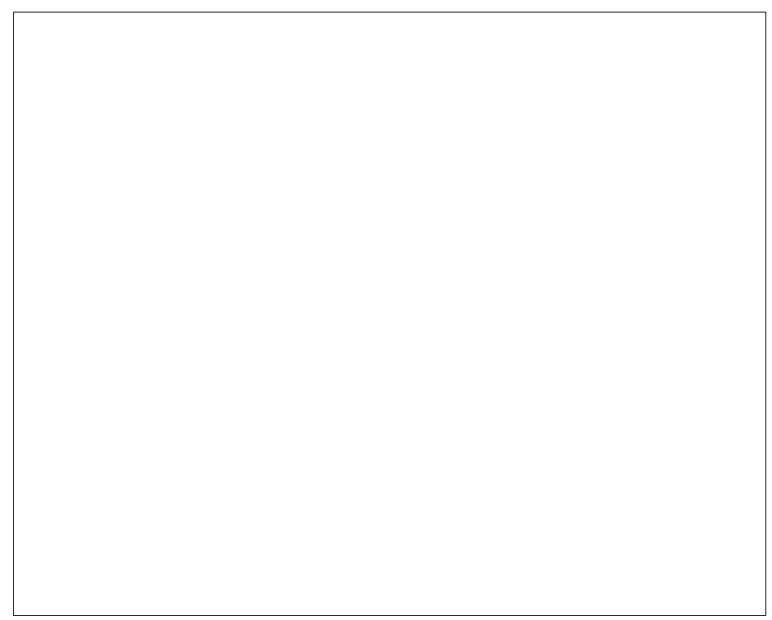

Wolfgang Mattheuer, Kain (1965), Halle (Saale), Stiftung Moritzburg. (c)Adagp, Paris, 2013.
Dans «Caïn » de Wolfgang Mattheuer (1965), le mythe du fratricide originel est actualisé par l'arrièreplan où l'on voit une ville moderne, mais il n'est pas rapporté à une actualisation précise. Le tableau peut être lu comme la représentation d'une constante de l'histoire de l'humanité - le meurtre, la guerre -, mais également comme une parabole de la guerre la plus actuelle en 1965, la guerre du Vietnam, et ce rapprochement a été fait dans la presse de RDA: Vietnam du Nord contre Vietnam du Sud, une guerre fratricide. Cette interprétation n'est pas en contradiction avec une lecture qui fait du tableau un symbole du conflit germano-allemand. Non plus Vietnam du Nord contre Vietnam du Sud, mais Allemagne de l'Ouest contre Allemagne de l'Est. Un commentateur de RDA s'est d'ailleurs engagé dans cette voie ${ }^{(23)}$ en disant qu'on peut voir dans ce tableau une allégorie du discours de la République fédérale sur les «frères et les sœurs » en RDA (die Brüder und Schwestern), qui est selon lui marqué au coin de la trahison et du revanchisme, et qui masque les préparatifs de guerre contre la RDA et le bloc socialiste. Malgré son ambivalence - chose encore très mal vue à l'époque - cette représentation peut être tirée dans le sens d'une critique du discours sur la pérennité de la nation allemande ${ }^{(24)}$.

Les départs à l'Ouest se multiplient dans les années $80 \mathrm{chez}$ les peintres qui ne font pas partie de l'establishment de la peinture de RDA. Mais le départ d'un artiste ne signifie pas qu'il disparaît complètement du paysage des arts plastiques en RDA. Le parcours du peintre de Dresde Ralf Kerbach en est un exemple: après son émigration en République fédérale en 1982, le contact avec ses collègues restés en RDA n'est pas rompu et les influences réciproques perdurent. En 1984, Kerbach peint le tableau qu'il appelle " Jumeau» ( Zwilling»). On y voit deux frères ligotés ensemble dos à dos, leurs crânes semblent soudés et les empêchent de se tourner l'un vers l'autre. L'une des têtes porte un bâillon qui l'empêche de parler, l'autre est entourée d'un barbelé qui

23 Dieter Gleisberg, «Wolfgang Mattheuer », in: Weggefährten. 25 Künstler der Deutschen Demokratischen Republik, Dresde, 1972, p. 230.

24 E. Gillen, Feindliche Brüder? (note 10), p. 341. De la même manière, dans le tableau de Bernhard Heisig «Beharrlichkeit des Vergessens », la banderole sur laquelle on lit: « Nous sommes pourtant tous frères et sœurs » («Wir sind doch alle Brüder und Schwestern [...] »), et qui est déployée de gauche à droite, c'est-à-dire d'ouest en est, marque une distance par rapport au discours sur la persistance de la nation allemande tel qu'il est tenu à l'Ouest, dans la mesure où ce discours peut être instrumentalisé et peut servir de paravent bien-pensant à une politique agressive vis-à-vis de la RDA. 
lui interdit de penser. Il s'agit clairement d'une métaphore de la dualité allemande telle que la vit un peintre passé récemment d'une Allemagne à l'autre. Cette représentation née à l'Ouest est reprise deux ans plus tard par un peintre resté à Dresde, Hubertus Giebe. Le centre de son tableau «La Résistance - pour Peter Weiss » (« Der Widerstand - für Peter Weiss ») est occupé par une réutilisation de cette figure jumelle: deux personnages entrecroisés et placés dos à dos se dirigent en sens inverse l'un de l'autre, ce sont les frères ennemis marchant sur des cadavres - une autre métaphore de la dualité allemande.

On peut voir à la $10^{\mathrm{e}}$ exposition nationale de Dresde le portrait d'une femme représentée debout devant la porte de Brandebourg vue du côté Est (Wolfgang Peuker: "A.P., geboren 1949 ", 1986). Née

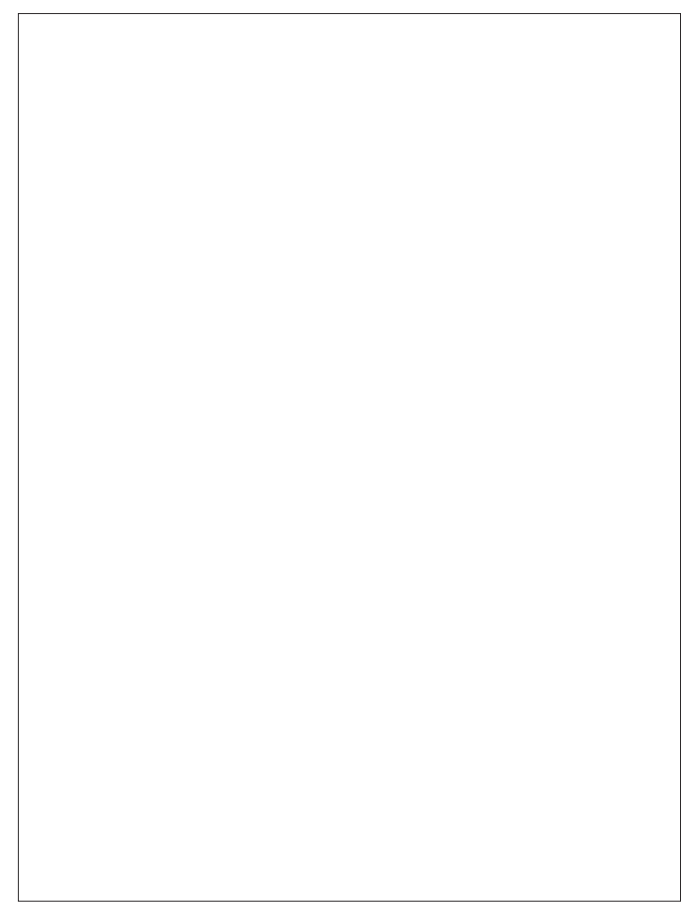

Ralf Kerbach, Zwilling (1984), Berlinische Galerie. (c) Adagp, Paris, 2013. en 1949 avec la RDA, cette femme (l'épouse du peintre) tourne le dos à Berlin-Ouest dont on aperçoit un monument à travers les colonnades de la porte de Brandebourg. Son regard mélancolique exprime une tristesse que l'on imagine en rapport avec la division de l'Allemagne, mais le mur lui-même reste hors champ, invisible, alors qu'il est pourtant présent à cet endroit avec une grande force symbolique. Les médias se cantonnent prudemment dans des appréciations formulées en termes généraux : «Le petit portrait 'A.P., née en 1949' met l'accent sur la psychologie du personnage et attire l'attention sur les liens entre évolution sociale et biographie individuelle " ${ }^{(25)}$. Un an plus tôt, W. Peuker avait peint un autoportrait qui le représentait en smoking devant une vue panoramique de Berlin traversée par le sillon du mur, mais ce tableau n'a pas eu les honneurs de l'exposition nationale de Dresde ${ }^{(26)}$.

Le mur de Berlin, couvert du côté Ouest d'une ceinture de fresques de style pop'art, est présent dans les œuvres de nombreux peintres et photographes de Berlin-Ouest pour lesquels il est un élément incontournable du cadre urbain. Dans la peinture de RDA, par contre, la représentation du mur est rare après l'année 1962 où certaines œuvres de commande ont mis en avant le caractère défensif du « rempart antifasciste ».

25 « Das kleine, psychologisch eindringliche Bildnis 'A.P., geboren 1949' macht auf die Verflechtungen zwischen gesellschaftlicher Entwicklung und individuellem Lebensgang aufmerksam. » K. M. KoBER, "Zunahme an Expressivität und Farbkraft» (note 17). 
Dans les années 1970, le tableau de Nuria Quevedo «Schwangere im Atelier am Pariser Platz ( (1972) fait figure d'exception. La situation d'exil dans laquelle vit le peintre n'est pas étrangère à sa vision d'une femme dont le cadre de vie est dominé par la coupure matérielle du mur et de son glacis, visibles par la fenêtre, mais qui est porteuse de l'espoir d'une vie nouvelle ${ }^{(27)}$. Konrad Knebel, connu pour ses paysages urbains, peint en 1977 «Straße mit Mauer» qui représente une rue barrée par le mur de Berlin surmonté d'un mirador. Mais ses tableaux retenus pour la $9^{\mathrm{e}}$ et la $10^{\mathrm{e}}$ exposition nationale de Dresde ne montrent que des rues dominées par d'immenses murs pare-feu. Dans les années 1980, on trouve encore peu d'œuvres dans lesquelles le mur de Berlin est montré de façon explicite et autrement que sous forme allusive. Dans « Berlin, Berlin III » de Trakia Wendisch, un couple de funambules évolue de nuit sur un fil tendu entre deux parties de la ville partagées par une rivière et un mur dont l'aspect évoque clairement la frontière fortifiée qui traverse Berlin, mais ce tableau ne sera peint qu'en $1990^{(28)}$.

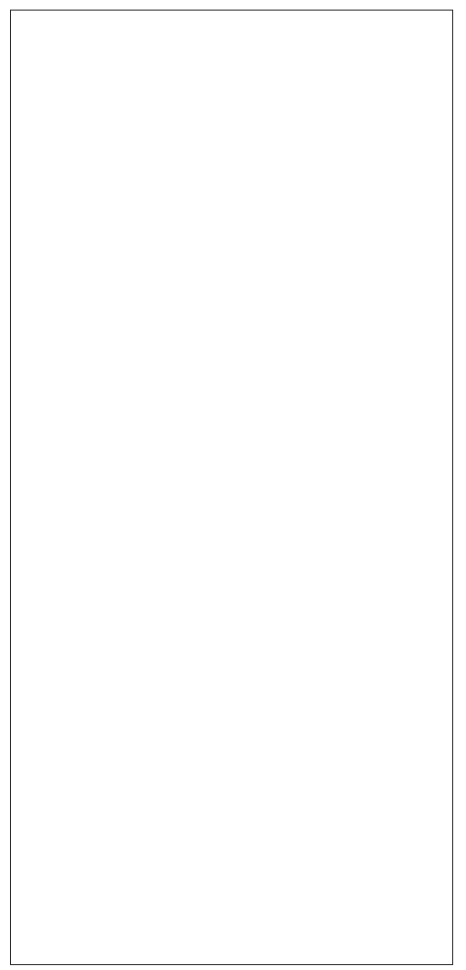

Klaus Killisch, Mann vor Mauer (1988), Stiftung Stadmuseum Berlin. (C) Adagp, Paris, 2013.
Il existe en revanche de nombreuses représentations métaphoriques du mur de Berlin, de nombreux tableaux barrés par un mur aveugle, ou au centre desquels se dresse un mur d'aspect banal: sans avoir la forme du mur de Berlin dans son état le plus élaboré, ces murs évoquent irrésistiblement celui-ci pour un public de RDA habitué à lire entre les lignes d'un texte ou à déchiffrer les allusions contenues dans une image. En 1988, Klaus Killisch avait peint et exposé à la galerie Eigen + Art de Leipzig « Mann vor Mauer »: un personnage en complet veston se heurte à un mur derrière lequel se profilent confusément un espace vide hérissé de quelques pieux et une silhouette humaine sans visage. En 1989, avant le déclenchement de la fuite en masse des citoyens de RDA par les ambassades de République fédérale et par la frontière austro-hongroise, Killisch avait peint "Mauerspringer », qui montre un homme enjambant au pas de course un muretin de briques. Plus que le muretin d'apparence anodine, c'est l'acte même de le franchir et le titre du tableau qui évoquent immanquablement "Le " mur. Bien d'autres tableaux qui ont trouvé place aux $9^{\mathrm{e}}$ et $10^{\mathrm{e}}$ expositions nationales de Dresde (1982-83, 1987-88) représentent des murs oppressants, angoissants qui expriment le syndrome d'enfermement dont souffre la population et l'obsession d'une improbable sortie.

27 Nuria Quevedo, originaire de Catalogne, vivait en exil en RDA depuis l'âge de 15 ans. Cf. aussi Sighard Gille, «Berliner Landschaft » (1976-1979).

28 Walter Libuda peint dans «Die Schleuse » (1987-88) une double rangée de murs contre lesquels sont appuyées des échelles destinées à les faire franchir, et un tunnel obscur qui débouche au pied du mur. 
Dans les années soixante, la seule évocation de l'existence du mur à travers l'expression picturale de la souffrance des individus séparés déclenchait les foudres des autorités. En 1965, un jeune peintre élève de Heisig, Hartwig Ebersbach, avait présenté à l'exposition régionale de Leipzig (7. Leipziger Bezirkskunstausstellung) un tableau intitulé «Selbstbildnis mit Freunden ». On y voyait le peintre nu entouré de deux personnages. Le peintre se mettait la main sur la bouche, signalant par ce geste l'obligation de se taire, la peur d'être entendu. La critique s'était dressée à l'époque contre l'expressivité crue - la nudité d'un corps déformé, scandaleuse pour l'époque - le centrage sur la souffrance et l'angoisse personnelles, l'absence de référence au « collectif». Cette souffrance s'éclaire d'un jour particulier quand on sait que les personnages représentés autour du peintre étaient des amis tous deux partis à l'Ouest (un ami peintre, et la propre sœur d'Ebersbach). La souffrance est celle du mur qui sépare les corps, la proximité illicite des corps séparés par le mur génère l'angoisse. Ebersbach a détruit le tableau après l'exposition, en réaction aux attaques massives de la critique qui en dénonçaient la subjectivité. Il n'en reste qu'une photo en noir et blanc qui rend mal compte de l'expressivité de l'œuvre. On peut toutefois s'en faire une idée à partir du tableau peint l'année suivante: "Brennender Mann », qui représente le peintre consumé de l'intérieur par un feu qui le dévore ${ }^{(29)}$.

La question de l'image de la nation dans les arts plastiques de RDA se double aujourd'hui d'une interrogation sur l'image des arts plastiques de RDA dans la nation réunifiée. Après l'unification, un débat virulent a mis en question l'appartenance des arts plastiques de RDA à la culture nationale allemande. Ce débat connu sous le nom de « querelle des images » (« Bilderstreit ») s'est aujourd'hui apaisé, mais il reste symptomatique de la difficulté à penser une culture nationale de l'après-guerre dans laquelle les déterminants politiques ne se substituent pas aux critères d'appréciation esthétiques. En 1990, il est encore possible de présenter la scène artistique est-allemande dans sa diversité sans jeter d'exclusives et sans provoquer de polémique. C'est ce que fait l'exposition de la collection Ludwig inaugurée à Cologne le 30 septembre 1990, quatre jours avant l'unification: Bilder aus Deutschland. Kunst der DDR aus der Sammlung Ludwig. Le titre de l'exposition indique clairement que l'art produit dans cette partie de l'Allemagne fait partie intégrante de la culture allemande. Eduard Beaucamp, critique d'art à la $F A Z$, écrit dans le catalogue: «Cet art est assez critique et assez fort pour interroger et pour enrichir de façon productive la scène artistique ouest-allemande ${ }^{\left({ }^{(30)}\right.}$.

Le climat change très vite. Deux ans plus tard, en 1992, a lieu la fusion des Académies des beaux-arts de Berlin-Ouest et de Berlin-Est (Akademie der Künste). Les membres de l'Académie issue de la RDA sont intégrés en bloc dans la nouvelle Académie unifiée. Cette fusion suscite un tollé, et 18 membres de l'Académie de l'Ouest démissionnent en signe de protestation, parmi lesquels Georg Baselitz, Gerhard Richter, Markus Lüpertz. Ils avancent l'argument selon lequel il ne peut pas y avoir d'art dans une dictature, pas même un art de mauvaise qualité. Dans le domaine artistique

29 Eckhart Gillen, Deutschlandbilder. Kunst aus einem geteilten Land, Cologne, Dumont, 1997, p. 548.

30 "Diese Kunst ist stark und kritisch genug, auch die westliche Szene produktiv herauszufordern und zu bereichern. » Eduard BeAucamp, « Dissidenten, Hofkünstler, Malerfürsten - Über die schwierige Wiedervereinigung deutscher Kunst», in: Evelyn WeIss (dir.), Bilder aus Deutschland. Eine Ausstellung des Museums Ludwig, Heidelberg, Braus, 1990, p. 34. 
il n'y a donc rien à unifier, et rien de ce qui a été produit en RDA ne mérite d'être intégré dans un patrimoine artistique national. Les deux galeries nationales de Berlin (Nationalgalerie et Neue Nationalgalerie) fusionnent elles aussi en 1993, et la réunion de leurs fonds donne lieu (en 1993-1994) à un nouvel accrochage de leurs collections d'œuvres allemandes contemporaines qui présente côte à côte des œuvres de l'Est et de l'Ouest: nouvelle levée de boucliers, où se font particulièrement entendre les peintres émigrés à l'Ouest avant 1989, les peintres marginaux de RDA, et une bonne partie des critiques artistiques. L'idée que l'on puisse réunifier les paysages artistiques de l'Est et de l'Ouest continue à choquer, la Nationalgalerie se voit accusée de cautionner l'art de la dictature, et les détracteurs demandent que l'on retire les œuvres des peintres les plus consacrés par la RDA, comme Heisig et Sitte. Le débat ne se situe plus sur le plan esthétique mais sur le plan politique, et là aussi on voit se profiler l'idée que cet art de l'Est est un non-art parce qu'il a volontairement ou non servi la dictature. L'addition d'un art et d'un non-art ne saurait donner un art national allemand.

Les années suivantes voient alterner les tentatives pour dépassionner le débat et les flambées de rejet passionné déclenchées par des expositions à Weimar, à Berlin, à Nuremberg. La démarche qui consiste à penser comme un tout l'évolution artistique dans les deux États allemands a mis du temps à se concrétiser. Elle a longtemps été entravée par la polémique offensive de la " querelle des images ». À partir du début du $\mathrm{XXI}^{\mathrm{e}}$ siècle, il est devenu possible de développer des comparaisons et de faire des mises en parallèle. Une tentative remarquable a été faite en ce sens par l'exposition L'art et la guerre froide. Positions allemandes 1949-1989 (Kunst und kalter Krieg. Deutsche Positionen 1945-89) qui a eu lieu en 2009-2010. Il est significatif que cette exposition ait été montée aux États-Unis, à Los Angeles, en collaboration avec un commissaire d'exposition allemand, et montrée à un public américain avant de l'être au public allemand. Il s'agit en effet d'une approche dépassionnée de l'histoire de l'art dans l'Allemagne divisée, qui cherchait à montrer qu'en dépit des contextes politiques très différents les artistes allemands de l'Est et de l'Ouest avaient plus de points communs qu'ils ne le pensaient ou qu'on ne le percevait généralement. Le choix des œuvres faisait apparaître qu'au moment de la chute du mur l'époque de la confrontation entre les artistes des deux parties de l'Allemagne était révolue, et que les arts plastiques de RDA dans les années 1980 étaient loin de présenter un visage homogène, surtout dans les milieux artistiques indépendants. Cette perspective étend au domaine artistique l'élaboration d'une histoire des deux Allemagnes après 1949 intégrant les « interdépendances asymétriques » qui, selon la formule de l'historien Christoph Kleßmann, ont caractérisé la situation particulière de l'Allemagne divisée.

Aujourd'hui, la Neue Nationalgalerie peut présenter sans soulever de polémique un nouvel accrochage « réunifié » de ses collections de l'Est et de l'Ouest. En 2011-2012, on peut y voir une exposition de ses œuvres de République fédérale et de RDA réunies sous le titre Der geteilte Himmel. Il n'est plus question d'exclure ou de rejeter en bloc les œuvres de RDA. On ne provoque plus de scandale quand on les intègre dans une vision contrastive de l'art des deux Allemagnes après 1945 qui insiste sur la perméabilité de la frontière entre le figuratif et l'abstrait malgré le «partage du ciel » ${ }^{(31)}$. Un tableau géant de Willi

31 L'exposition présente sous cet angle l'œuvre de Heinrich Ehmsen et la réception de Picasso chez Metzkes, Strawalde et Tübke. 
Sitte qui évoque l'échec du soulèvement communiste à Leuna en 1921, présenté au même endroit en 1994, était dénoncé comme indésirable dans le temple de la vraie peinture et de l'art libre. Aujourd'hui, il est lu comme le «Guernica » de la peinture de RDA ${ }^{(32)} \ldots$ Mais la peinture de RDA qui ne se prête pas à ces rapprochements, celle qui prend pour thèmes le monde ouvrier et la vie quotidienne, est pour l'instant encore absente de la confrontation $^{(33)}$. La «nationalisation » des arts plastiques de RDA se fait pas à pas.

\title{
Résumé
}

Le régime de RDA récuse à partir des années 1970 la référence à la nation allemande et se réclame d'une autre nation. Cette évolution suscite dans les arts plastiques un questionnement autour des représentations de la nation. L'interrogation présente dans la peinture qui prend pour objet le mouvement ouvrier et le national-socialisme s'exprime également à travers les représentations métaphoriques de la division allemande, qui inclut l'image souvent indirecte de la frontière. La question de l'image de la nation se double après l'unification d'un débat sur la place des arts plastiques de RDA dans l'art national.

\section{Zusammenfassung}

Im Zuge der Abgrenzungspolitik verwarf die DDR die Vorstellung einer einheitlichen deutschen Nation und berief sich auf einen neuen Begriff der Nation. Aufgrund dieser Entwicklung hinterfragten bildende Künstler der DDR die Darstellungen der Nation in der Historienmalerei, namentlich zu den Schwerpunkten Arbeiterbewegung und Nationalsozialismus, und setzten sich in metaphorischen Bildern mit dem Problemfeld von Spaltung und Gemeinsamkeiten, in oft verhüllter Form auch mit der innerdeutschen Grenze auseinander. Im wiedervereinigten Deutschland entwickelte sich ein heftiger Streit um die Integration der bildenden Künste der DDR in eine gemeinsame nationale Tradition.

\begin{abstract}
The GDR refused, since the 70s, to be referred to a German nation and claimed to be a separate nation. In the art world, this evolution has given rise to a questioning centered around representation of the nation. The questioning present in paintings whose themes are the labour movement and the National Socialism, finds its expression equally by means of metaphorical representations of the german divide which include the image, often indirect, of the frontier. After unification, a debate takes place about the role that art from the GDR should play in the national art tradition.
\end{abstract}

32 Peter Richter, « Die geteilte Hölle », Frankfurter Allgemeine Zeitung, 13.11.2011.

33 La peinture du monde ouvrier et de la vie quotidienne est largement représentée dans l'exposition Abschied von Ikarus (Weimar, 2012-2013) qui présente les figures de l'utopie et de son abandon dans la peinture de RDA, mais la perspective n'est pas contrastive. Néanmoins, cette exposition est conçue comme une incitation à faire sortir la peinture de RDA des réserves des musées et à lui donner une place dans les présentations permanentes. 